\title{
Effect of high-LET Fe-ion beam irradiation on mutation induction in Arabidopsis thaliana
}

\author{
Yusuke Kazama ${ }^{1 \dagger}$, Tomonari Hirano ${ }^{1 \dagger}$, Kiyoshi Nishihara ${ }^{2}$, Sumie Ohbu ${ }^{2}$, \\ Yuki Shirakawa ${ }^{2}$ and Tomoko Abe ${ }^{1,2 *}$ \\ ${ }^{1}$ RIKEN Innovation Center, 2-1 Hirosawa, Wako, Saitama 351-0198, Japan \\ ${ }^{2}$ RIKEN Nishina Center, 2-1 Hirosawa, Wako, Saitama 351-0198, Japan
}

(Received 31 May 2013, accepted 15 July 2013)

\begin{abstract}
Heavy-ion beams are powerful mutagens. They cause a broad spectrum of mutation phenotypes with high efficiency even at low irradiation doses and short irradiation times. These mutagenic effects are due to dense ionisation in a localised region along the ion particle path. Linear energy transfer $\left(\mathrm{LET} ; \mathrm{keV} \cdot \mu \mathrm{m}^{-1}\right.$ ), which represents the degree of locally deposited energy, is an important parameter in heavy-ion mutagenesis. For high LET radiation above $290 \mathrm{keV} \cdot \mu \mathrm{m}^{-1}$, however, neither the mutation frequency nor the molecular nature of the mutations has been fully characterised. In this study, we investigated the effect of Fe-ion beams with an LET of $640 \mathrm{keV} \cdot \mu \mathrm{m}^{-1}$ on both the mutation frequency and the molecular nature of the mutations. Screening of well-characterised mutants ( $h y$ and $g l$ ) revealed that the mutation frequency was lower than any other ion species with low LET. We investigated the resulting mutations in the 4 identified mutants. Three mutants were examined by employing PCR-based methods, one of which had 2-bp deletion, another had $178 \mathrm{bp}$ of tandemly duplication, and other one had complicated chromosomal rearrangements with variable deletions in size at breakpoints. We also detected large deletions in the other mutant by using array comparative genomic hybridisation. From the results of the analysis of the breakpoints and junctions of the detected deletions, it was revealed that the mutants harboured chromosomal rearrangements in their genomes. These results indicate that Fe-ion irradiation tends to cause complex mutations with low efficiency. We conclude that Fe-ion irradiation could be useful for inducing chromosomal rearrangements or large deletions.
\end{abstract}

Key words: Arabidopsis thaliana, array comparative genomic hybridisation (array CGH), heavy-ion beam, linear energy transfer (LET), mutation induction

\section{INTRODUCTION}

Heavy-ion beams comprise accelerated heavy ions that are produced by using accelerators such as cyclotrons or synchrotrons. They have been accepted as an efficient technology for the mutation breeding of plants and microbes. Heavy-ion irradiation induces a broad spectrum of mutation phenotypes even at low irradiation doses and for short irradiation times (Tanaka et al., 2010; Abe et al., 2012). Heavy-ion irradiation has also been accepted as a powerful mutagen for both forward and reverse genetics. Therefore, studies on biological processes in plants and microbes have been advanced by using heavy-ion irradiation (Hase et al., 2000, 2006; Shitsukawa et al., 2007; Shimada et al., 2009; Yasui et al., 2011;

\footnotetext{
Edited by Koji Murai

* Corresponding author. E-mail: tomoabe@riken.jp

$\uparrow$ These authors equally contributed to this study
}

Fujita et al., 2012; Sasaki et al., 2012; Ishikawa et al., 2012; Kazama et al., 2013; Hirano et al., 2013; Ma et al., 2013).

A noted physical characteristic of heavy-ion beams is that the accelerated particles densely deposit their energy in a localised region along the particle path. Therefore, heavy-ion beams are called high linear energy transfer $\left(\mathrm{LET} ; \mathrm{keV} \cdot \mu \mathrm{m}^{-1}\right.$ ) irradiation, as opposed to low LET irradiation from X-rays and $\gamma$-rays. The LET of $\gamma$-rays is 0.2 $\mathrm{keV} \cdot \mu \mathrm{m}^{-1}$, whereas the LET of heavy-ion beams can be altered by selecting the ion species or controlling its velocity. It is well known that high-LET radiation shows stronger biological effects than low-LET radiation (Yatagai, 2004; Tanaka et al., 2010). We have studied the effect of LETs by using the model plant Arabidopsis thaliana. When the albino mutant incidence was investigated in the $\mathrm{M}_{2}$ generation after irradiation treatment with heavy-ion beams with LETs ranging from 22.5 to 640 
$\mathrm{keV} \cdot \mu \mathrm{m}^{-1}$, the most effective LET was found to be 30.0 $\mathrm{keV} \cdot \mu \mathrm{m}^{-1}$ (Kazama et al., 2008). This irradiation treatment showed similar mutagenic efficiency to that of ethyl methanesulphonate (Koornneef et al., 1982; Kazama et al., 2011). The LET of $30.0 \mathrm{keV} \cdot \mu \mathrm{m}^{-1}$ was also efficient for causing a high mutation frequency in the $M_{1}$ generation (Kazama et al., 2012). This highly efficient LET was designated as LETmax. The value of the LET also affects the size of the induced deletion: it becomes large with increasing LET (Shikazono et al., 2005; Kazama et al., 2011; Hirano et al., 2012). Taken together, it is possible to select the LET value depending on the experimental purpose or target genes. LETmax is suitable for inducing single gene mutations, because it provides a high mutation frequency and mainly causes deletions with the size range of 1-53 bp (Kazama et al., 2011). LET at $290 \mathrm{keV} \cdot \mu \mathrm{m}^{-1}$ is useful for disrupting tandemly arrayed multiple genes, since it causes large deletions, the extent of which disrupt more than 2 tandemly arrayed genes (Hirano et al., 2012).

Herein, we focus on the mutagenic effect of $\mathrm{Fe}$ ions, which have the highest LET $\left(640 \mathrm{keV} \cdot \mu \mathrm{m}^{-1}\right)$ among the commonly used ion species at RIKEN. In a study on the microbe Mesorhizobium loti, Fe ions showed a tendency to induce larger deletions with higher mutation frequencies than carbon ions at $23 \mathrm{keV} \cdot \mu \mathrm{m}^{-1}$ (Ichida et al., 2008). On the other hand, knowledge of the effect of heavy-ion beams with higher LET regions, such as $640 \mathrm{keV} \cdot \mu \mathrm{m}^{-1}$, is still limited for plants. In this study, we investigated the mutation frequencies and sizes of mutated DNA regions in A. thaliana after irradiation with Fe ions. We also adopted an array-comparative genomic hybridisation (array CGH) analysis to investigate the effect of mutation induction at the whole genome level.

\section{MATERIALS AND METHODS}

Irradiation treatment Dry seeds of A. thaliana ecotype Columbia (Col-0) were packed in a plastic bag to obtain a monolayer of seeds. The seeds were irradiated with ${ }^{56} \mathrm{Fe}^{24+}$ ions at a dose of 50 Gy by using the E5 beam line in the RIKEN RI-beam factory. The ions were accelerated up to $90 \mathrm{MeV} /$ nucleon, at which the LET value of the ${ }^{56} \mathrm{Fe}^{24+}$ ions was $640 \mathrm{keV} \cdot \mu \mathrm{m}^{-1}$. The LET value was calculated behind the seeds. The irradiated $M_{1}$ seeds were surface-sterilised by dipping them in $1 \%$ sodium hypochlorite for $10 \mathrm{~min}$. Following this, they were washed 5 times with sterilised water, and then incubated on $0.7 \%$ agar-containing Murashige and Skoog (MS) medium that was supplemented with MS vitamins and $3 \%$ sucrose at $4^{\circ} \mathrm{C}$ in the dark for $4 \mathrm{~d}$ to induce vernalisation. The $\mathrm{M}_{2}$ seeds were collected as described previously (Kazama et al., 2011; Hirano et al., 2012). In short, eleven seedlings were grown in a plastic tray, from which $\mathrm{M}_{2}$ seeds of all plants were corrected and were treated as one batch.

Mutant screening and identification of mutated genes At least $110 \mathrm{M}_{2}$ seeds per batch were sown, and germinating plants were used for mutant screening. From the $\mathrm{M}_{2}$ generation, hy and $g l$ mutants were screened, whose phenotypes were long-hypocotyl (Koornneef et al., 1980) and non trichome (Walker et al., 1999), respectively. The putative responsible genes of $h y$ and $g l$ mutants (HY1, HY2, HY3, HY4, and HY5 for hy mutants; GL1, GL2, and TTG1 for $g l$ mutants) were amplified by performing PCR, and then the amplified fragments were sequenced as described previously (Kazama et al., 2011; Hirano et al., 2012). When the whole region or a part of the coding region of the putative responsible genes could not be amplified, flanking sequence analysis by using TAIL-PCR was performed (Liu et al., 1995). Because of the limited number of $h y$ and $g l$ mutants that were identified, we also screened 2 morphological mutants, brevipedicellus $(b p)$, whose phenotype was downward-pointing flowers (Venglat et al., 2002), and narrow leaves ( $\mathrm{nl}$ ) (Kim et al., 2002; Horiguchi et al., 2005). Then, we analysed their putative responsible genes ( $B P$ for $b p$ mutant; $A N$, AN3, AtGRF5 for $n l$ muant). The $\mathrm{M}_{3}$ seeds of the mutants were harvested and the phenotypes of the $M_{3}$ plants were analysed to confirm whether the phenotypes of the mutants were inherited.

Array CGH analysis We requested support from Roche NimbleGen Inc. (Madison, WI, USA) for the generation of custom oligonucleotide arrays. The whole genome sequence of $A$. thaliana (TAIR8, http://www.arabidopsis. org/) was tiled with oligonucleotides that started every 50 bp. Oligonucleotide probes were designed to represent the non-repetitive DNA sequence. The oligonucleotides were designed to be of variable length and adjusted to match a target melting temperature of $76^{\circ} \mathrm{C}$. Probe lengths were constrained to a minimum of 50 and a maximum of $75 \mathrm{bp}$. The DNA of the wild-type and the mutant (Fe-148-pg1) was isolated by using a Nucleon PhytoPure kit (GE Healthcare UK Ltd, Buckinghamshire, England). The DNA was labelled as described previously (Lucito et al., 2003). The DNA of the wild type and that of the mutant (Fe-148-pg1) were labelled with Cy5 and Cy3, respectively. Hybridisation, washing, and scanning were conducted by Roche NimbleGen. Fluorescence intensity raw data were obtained from scanned images of the oligonucleotide tiling arrays by using NimbleScan 2.4 extraction software (Roche NimbleGen Inc.). For each spot on the array, $\log _{2}$ ratios of the Cy3-labelled sample (Fe148pg1 mutant) vs. the Cy5-reference (wild-type) were calculated.

The sequence positions that are represented in the present study were based on The Arabidopsis Information Resource (TAIR 10; http://arabidopsis.org). 


\section{RESULTS}

Mutant screening The $h y$ and $g l$ mutants were screened in the $\mathrm{M}_{2}$ generation after Fe-ion irradiation at a dose of $50 \mathrm{~Gy}$, for which the highest frequencies of albino mutants was observed in the dose range 5-150 Gy (Kazama et al., 2008). The methods of screening and calculation of mutation frequencies were the same as those of previous studies (Kazama et al., 2011; Hirano et al., 2012). The mutation frequencies of the $h y$ and $g l$ mutants for Fe-ion irradiation were $0.13 \%$ and $0.03 \%$, respectively (Table 1). These mutation frequencies were lower than that for any other ions that were previously tested (Kazama et al., 2011; Hirano et al., 2012). Because of the low mutation frequencies for Fe-ion irradiation, we also isolated one $b p$ mutant (Venglat et al., 2002) and one $n l$ mutant (Kim et al., 2002; Horiguchi et al., 2005) from the $\mathrm{M}_{2}$ generation, and included them for the characterisation of the DNA mutations.
Characterisation of DNA mutations induced by using Fe-ion irradiation To investigate the structure of mutated DNA in the isolated mutants that was caused by carrying out $\mathrm{Fe}$-ion irradiation, DNA from the isolated mutants was subjected to PCR and sequencing analyses by using primers specific for the genes that were responsible for the $h y, g l, b p$, and $n l$ phenotypes. From the genome of the hy mutant (Fe-87-hy1), fragments having similar sizes compared to that of the wild type were amplified by performing PCR. Sequencing analysis revealed that the mutant harboured a 2-bp deletion in the second exon of the gene HY3 (Fig. 1A). All of the hy mutants were isolated from the same batch and were thought to have originated from same $\mathrm{M}_{1}$ plants. To ascertain that all mutants classified in the same mutant line had an identical DNA mutation, the hy mutants were confirmed by PCR and sequencing. Since all of the hy mutants harboured 2-bp deletion in the HY3 gene, we considered the hy mutants as one mutant line (Fe-87-

Table 1. Number of hy and $g l$ mutants induced by Fe-ion beam

\begin{tabular}{|c|c|c|c|c|}
\hline \multirow{2}{*}{ Ion species } & \multicolumn{2}{|c|}{ Total number of plants } & \multirow{2}{*}{$\begin{array}{c}\text { hy mutant } \\
\text { (mutation frequency*) }\end{array}$} & \multirow{2}{*}{$\begin{array}{c}g l \text { mutant } \\
\text { (mutation frequency*) }\end{array}$} \\
\hline & $\mathrm{M}_{1}$ & $\mathrm{M}_{2}$ & & \\
\hline${ }^{56} \mathrm{Fe}^{24+}$ & 2,893 & 29,176 & $4(0.13)$ & $1(0.03)$ \\
\hline
\end{tabular}

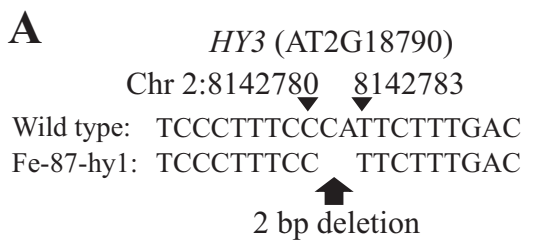

C

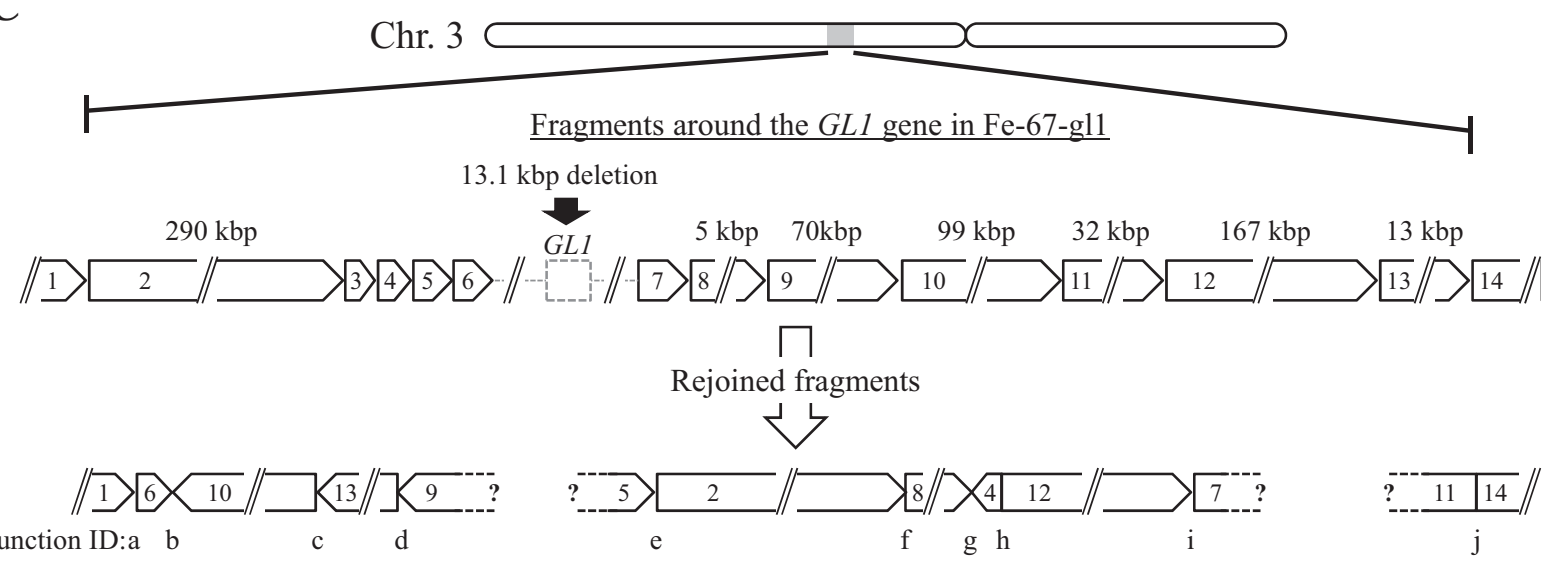

Fig. 1. Mutations identified in the mutants that were induced by using an Fe-ion beam. The sequences of the mutated genes in the mutants were compared to that of the wild type: the mutant line of Fe-87-hy1 harboured a deletion in the HY3 gene (A) and tandem duplication was detected in the $A N$ gene of Fe-88-nl1 (B). (C) Schematic representation of the rearrangements in Fe-67-gl1. The fragments around the GL1 gene in chromosome (Chr) 3, which were estimated by the break points that are represented in Supplementary Fig. S1, are shown with the boxes given the direction. The rejoined fragments were based on the junctions that are represented in Supplementary Fig. S2. 
hy1). PCR analysis of the $n l$ mutant (Fe-88-nl1) showed amplification of a fragment that was larger than the wild type. Sequencing analysis revealed that the Fe-88-nl1 mutant had a 178-bp duplication in the gene AN3 (Fig. 1B). Both ends of the 178-bp duplication had a common 5-bp sequence (GGTAA).

Complex rearrangement detected in the Fe-67-gl1 mutant In the Fe-67-gl1 mutant, a 13-kbp region that covered the entire sequence of GL1 could not be amplified
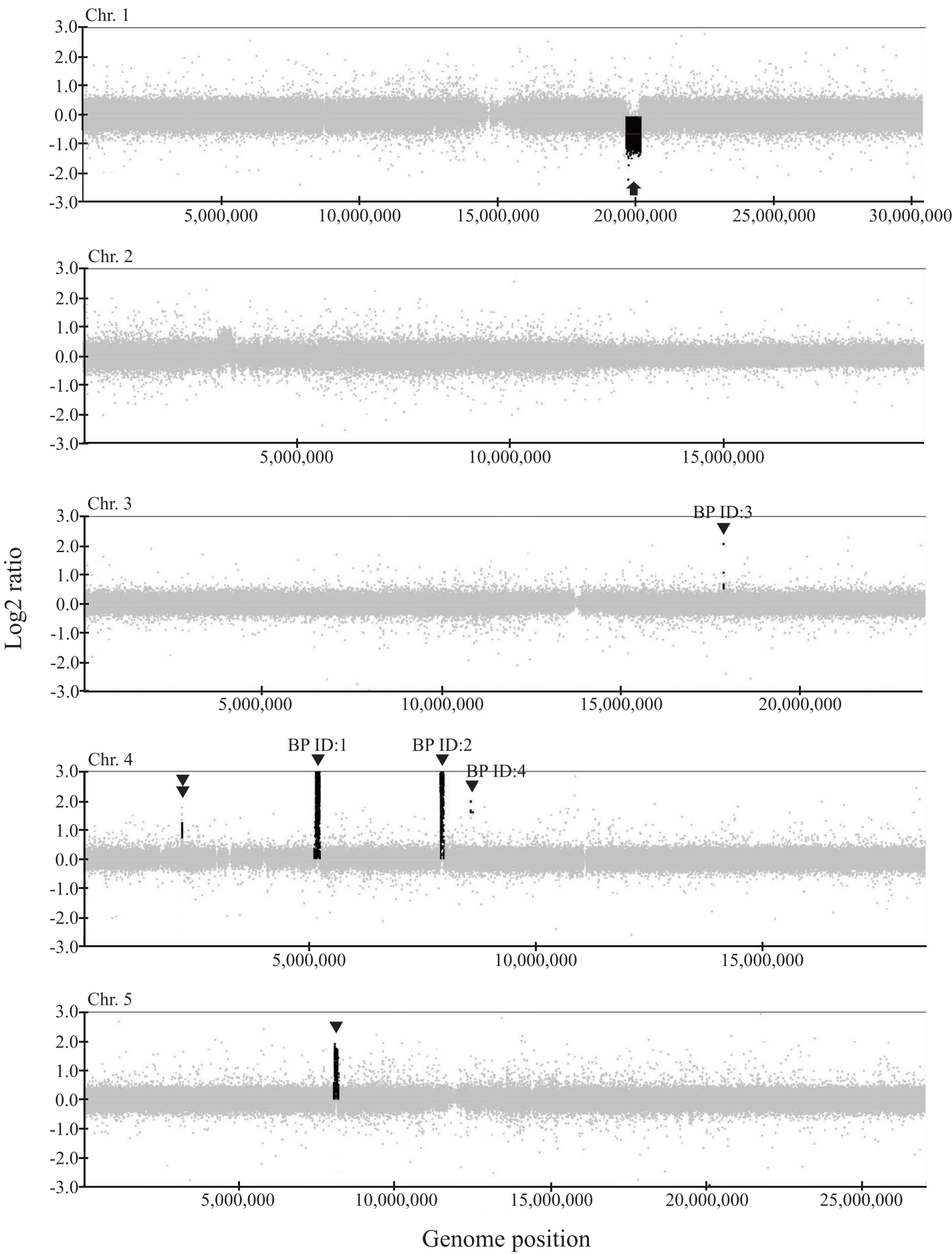

Fig. 2. Detection of mutations in the mutant line of Fe-148-pg1 by employing array CGH. The vertical and horizontal axes indicate the $\log _{2}$ ratio and positions of the probes in each chromosome (Chr), respectively. The arrowheads and arrows represent the deleted and duplicated areas, respectively. The double arrowhead indicates the area including two deletions that are closely positioned. The break point (BP) IDs written above the arrowheads correspond to those in Table 2. 
by performing PCR. Because no fragments were amplified by using primer sets that were designed for both ends of this $13-\mathrm{kbp}$ region, we expected that some chromosomal rearrangements had taken place. To identify the junction sequences of the rearrangements, we designed primers for TAIL-PCR. When a fragment was amplified by performing TAIL-PCR, it was sequenced. Then, another TAIL-PCR was performed to identify the next junction. We continued to perform TAIL-PCR until no fragment was amplified, which was probably due to genomic repetitiveness or extensively low G/C content. All of the detected junctions in the Fe-67-gl1 mutant are shown in Fig. 1C. A 689-kbp chromosomal region in chromosome 3, including the GL1 gene, was divided into at least 14 fragments. The 14 fragments may have been rearranged without the $13-\mathrm{kbp}$ fragment. All of the possible breakpoints that were detected contained deletions ranging from 2 to $13,139 \mathrm{bp}$, and none had duplications (Supplementary Fig. S1). Of the detected junctions, 6 had short regions with sequence homology (microhomology), whereas 5 had inserted DNA fragments, which are termed filler DNA (Gorbunova and Levy, 1999). There was no junction that simply rejoined the broken ends (Supplementary Fig. S2). The 689-kbp chromosomal region in chromosome 3 also contained 447-bp fragment derived from chromosome 2 (Supplementary Fig. S2, Junction ID: g). We performed PCR to test whether the corresponding fragment on the chromosome 2 was amplified in the Fe-67-gl1 mutant or not. As a result, a fragment having the same size as that in the wild type were amplified from chromosome 2 of the mutant, indicating that the 447-bp fragment was dupli- cated in the genome of the Fe-67-gl1 mutant.

Genomic evaluation of mutations in Fe-148-pg1 by using array CGH From phenotypic characterisation, Fe-148-pg1 was expected to be a mutant of $b p$ (Venglat et al., 2002). When PCR was performed by using primer sets that were designed around the $B P$ gene, no fragments were amplified from the Fe-148-pg1 mutant. We then designed primer sets on the neighbouring regions of $B P$. The PCR results indicated that the expected deletion in Fe-148-pg1 was over $80 \mathrm{kbp}$ (data not shown). Array CGH is an effective method for detecting such a large deletion (Nagano et al., 2008; Yu et al., 2011). Moreover, array CGH can be used to detect deletions and duplications at the whole genome level. For array CGH analysis, we created an original array in which each probe was designed at every $50 \mathrm{bp}$ (see MATERIALS AND METHODS). Candidate copy number variations were identified by finding more than 4 consecutive probes with $\log _{2}$ ratios that were greater than \pm 1.0 (Yu et al., 2011). Thus, we expected that our array was able to detect deletions that were more than $200 \mathrm{bp}$ at the whole genome level. DNA from the wild-type Columbia (Col-0) plant and the bulk of the $\mathrm{M}_{3}$ plants of the Fe-148-pg1 mutant were labelled with Cy5 and Cy3, respectively, and then hybridised to the array (see MATERIALS AND METHODS). The results are shown in Fig. 2 and Table 2. The candidate deletions were confirmed by performing PCR. For the PCR test, 7 individual $\mathrm{M}_{3}$ plants were tested to detect both homozygous and heterozygous deletions. When some of the plants showed amplifica-

Table 2. List of deletion peaks estimated by array CGH in Fe-148-pg1

\begin{tabular}{|c|c|c|c|c|c|c|}
\hline \multicolumn{3}{|c|}{ Peak area } & \multirow{2}{*}{ Signal* } & \multirow{2}{*}{$\begin{array}{c}\text { Estimated } \\
\text { deletion size }\end{array}$} & \multirow{2}{*}{$\begin{array}{l}\text { Detected deletion } \\
\text { (Break point ID) }\end{array}$} & \multirow{2}{*}{$\begin{array}{c}\text { Genetic } \\
\text { homogenity in } \mathrm{M}_{3}\end{array}$} \\
\hline Chr. & Start & End & & & & \\
\hline 4 & $5,129,711$ & $5,217,779$ & $1.500-6.097$ & 88,069 & (BP ID: 1$)$ & homogenous \\
\hline 4 & $7,875,508$ & $7,956,615$ & $3.375-6.047$ & 81,108 & $\bigcirc$ (BP ID: 2$)$ & homogenous \\
\hline 1 & $17,030,769$ & $17,031,235$ & 2.289 & 467 & - & - \\
\hline 3 & $17,831,368$ & $17,831,817$ & 2.076 & 450 & (BP ID: 3 ) & homogenous \\
\hline 4 & $8,550,408$ & $8,550,671$ & 1.992 & 264 & (BP ID: 4) & homogenous \\
\hline 5 & $8,060,900$ & $8,145,715$ & $1.744-1.930$ & 84,816 & $\bigcirc$ & heterogeneous \\
\hline 4 & $2,179,908$ & $2,180,757$ & 1.777 & 850 & - & - \\
\hline 4 & $2,177,558$ & $2,178,371$ & 1.767 & 814 & - & - \\
\hline 4 & $2,185,508$ & $2,186,230$ & 1.743 & 723 & $\bigcirc$ & heterogeneous \\
\hline 4 & $2,181,208$ & $2,181,676$ & 1.209 & 469 & $\bigcirc$ & heterogeneous \\
\hline 5 & $13,568,975$ & $13,569,992$ & 1.103 & 1,018 & - & - \\
\hline 4 & $2,178,908$ & $2,179,371$ & 1.082 & 464 & - & - \\
\hline 4 & $2,183,558$ & $2,184,029$ & 1.037 & 472 & - & - \\
\hline 5 & $20,664,928$ & $20,665,238$ & 1.028 & 311 & - & - \\
\hline 2 & $3,314,909$ & $3,315,477$ & 1.011 & 569 & - & - \\
\hline
\end{tabular}

The peaks with high signals $(>1.0)$ were listed.

* When several peaks were detected in naboring area, we combined the peaks and showed the range of signal values. 
tion in the region of the candidate deletion, the candidate deletion was determined as being heterozygous in the $\mathrm{M}_{2}$ generation. Finally, we detected 7 deletions and 1 candidate duplication (we could not confirm the duplication by performing PCR) (Table 2 and Fig. 2). Candidate deletions that were supported by the higher $\log _{2}$ ratio tended to be homozygous. On the other hand, candidate deletions that were supported by $\log _{2}$ ratios lower than 1.209 were false positives. We also tested 4 additional candidates that were supported by $\log _{2}$ ratios lower than 1.0: they were also false positives (data not shown). From the results, it is suggested that candidates having $\log _{2}$ ratios of over 1.2 should be considered as possible candidates in this array platform. We attempted to detect the breakpoints and junctions of 4 homozygous deletions by performing TAIL-PCR (BP ID: 1-4 in Table 2 and Fig. 3). The breakpoints that were detected revealed that the actual size of the deleted regions ranged from 7 to $90,737 \mathrm{bp}$. In the 8 junctions that were detected, 4 had micro-homology (the upstream junction of BP ID: 2, and the downstream junctions of BP ID: $1, \mathrm{BP}$ ID: 2, and BP ID: 3 ) and 1 had filler DNA (the upstream junction of BP ID: 1). There was no simple deletion in which both the up and down stream ends of a deletion were rejoined directly. One of the break ends in BP ID: 3 was rejoined to the candidate region of duplication in chromosome 1 (Fig. 3), which suggests that a duplicated fragment was part of the rearrangement.

\section{DISCUSSION}

Low mutation frequencies in Fe-ion irradiation Our results revealed that mutation frequencies in the $\mathrm{Fe}$ ion-irradiated $A$. thaliana were lower than those in the other ion-irradiated specimens (C ion with LET of 22.5 or $30.0 \mathrm{keV} \cdot \mu \mathrm{m}^{-1}$, Kazama et al., 2011; $\mathrm{C}$ ion and Ar ion with LET of $290 \mathrm{keV} \cdot \mu \mathrm{m}^{-1}$, Hirano et al., 2012), when the incidences of the $h y$ and $g l$ mutants were calculated for the $\mathrm{M}_{2}$ generation. Low mutation frequencies for Fe-ion irradiation were also observed when the incidence of albino mutants was determined (Kazama et al., 2008). These outcomes are likely due to the low number of irradiated $\mathrm{Fe}$ ions. Because the dose is proportional to the product of the LET multiplied by the number of ion particles, the number of Fe-ion particles $\left(640 \mathrm{keV} \cdot \mu \mathrm{m}^{-1}\right)$ is lower than that of the other ion particles $(22.5,30.0$, or $290 \mathrm{keV} \cdot \mu \mathrm{m}^{-1}$ ) at the same dose. Thus, irradiation with Fe ions requires a higher dose than irradiation with other ions to obtain the same particle number. For example, with the same particle number for Ar ions at its most effective dose (50 Gy), the dose of Fe-ion irradiation reached about 110 Gy. However, a severe lethal effect was observed at this dose for Fe-ion irradiation (Kazama et al., 2008). Therefore, such high-LET radiation could not result in the high mutation frequency. The low mutation frequency for Fe-ion irradiation could also be explained by the proportion of induced chromosomal rearrangements. It has been reported that large deletions or rearrangements cannot be transmitted to the next generation because they include genes that are essential for gamete development or viability (Naito et al., 2005). We previously observed that the frequency of large deletions and rearrangements increases as the LET values increase (Kazama et al., 2011; Hirano et al., 2012). We also observed that the transmissibility of the mutations also decreases as the LET values of the radiation increase (Kazama et al., 2012). It is possible that Fe-ion irradiation could induce chromosomal rearrangements with high efficiency, thereby resulting in a low mutation frequency in the $\mathrm{M}_{2}$ generation.

Complex rearrangement induced by Fe-ion irradiation Although the number of analysed mutants was very small, we determined complex rearrangements in the 2 of the 4 mutants that we analysed. One had intrachromosomal rearrangements with a large deletion (Fe67-gl1; Fig. 1C) and the other had interchromosomal rearrangements with 3 large deletions (Fe-148-pg1; Fig. 3). It is likely that these complex rearrangements were typical mutations that are caused by such high-LET irradiation. Data from both Monte Carlo calculations and experimental measurements have revealed that lowLET radiation-induced double strand breaks (DSBs) are distributed more uniformly along the DNA, whereas a much larger proportion of high-LET radiation-induced DSBs are distributed locally and densely (Fakir et al., 2006; Alloni et al., 2010). Moreover, complex DSBs are difficult to repair (Blocher, 1988; Pastwa et al., 2003). Therefore, it is reasonable to assume that the highly complex rearrangements that were detected in this study may have been produced because of repair or misrepair of the densely distributed DSBs. Large deletions could emerge when the fragmented DNA that is produced by clustering DSBs is lost. Complex intrachromosomal rearrangements could be produced when fragmented DNA is randomly rejoined with each other. Such misrepairs and complex rearrangements may also contribute to the loss of transmissibility of the mutated genes (see above).

Most of all, the breakpoints that were detected in this study had deletions whose sizes varied from 2 to 90,737 bp (Fig. 3; Supplementary Figs. S1 and S2). Shikazono et al. (2005) reported that electrons cause duplications at breakpoints, whereas heavy-ion beams do not. These observations are well supported by our results. The proportion of deletions with sizes from $100 \mathrm{bp}$ to $1 \mathrm{kbp}$ to the total number of deletions that were detected in Fe-ion irradiation was high $(31 \% ; 4$ deletions of the identified 13 ones, see Figs. 1A and 3; Supplementary Fig. S1) in comparison to deletions that are induced by $\gamma$-rays $(0 \%$, Morita et al., 2009) and heavy ions with lower LETs $(0 \%$ 

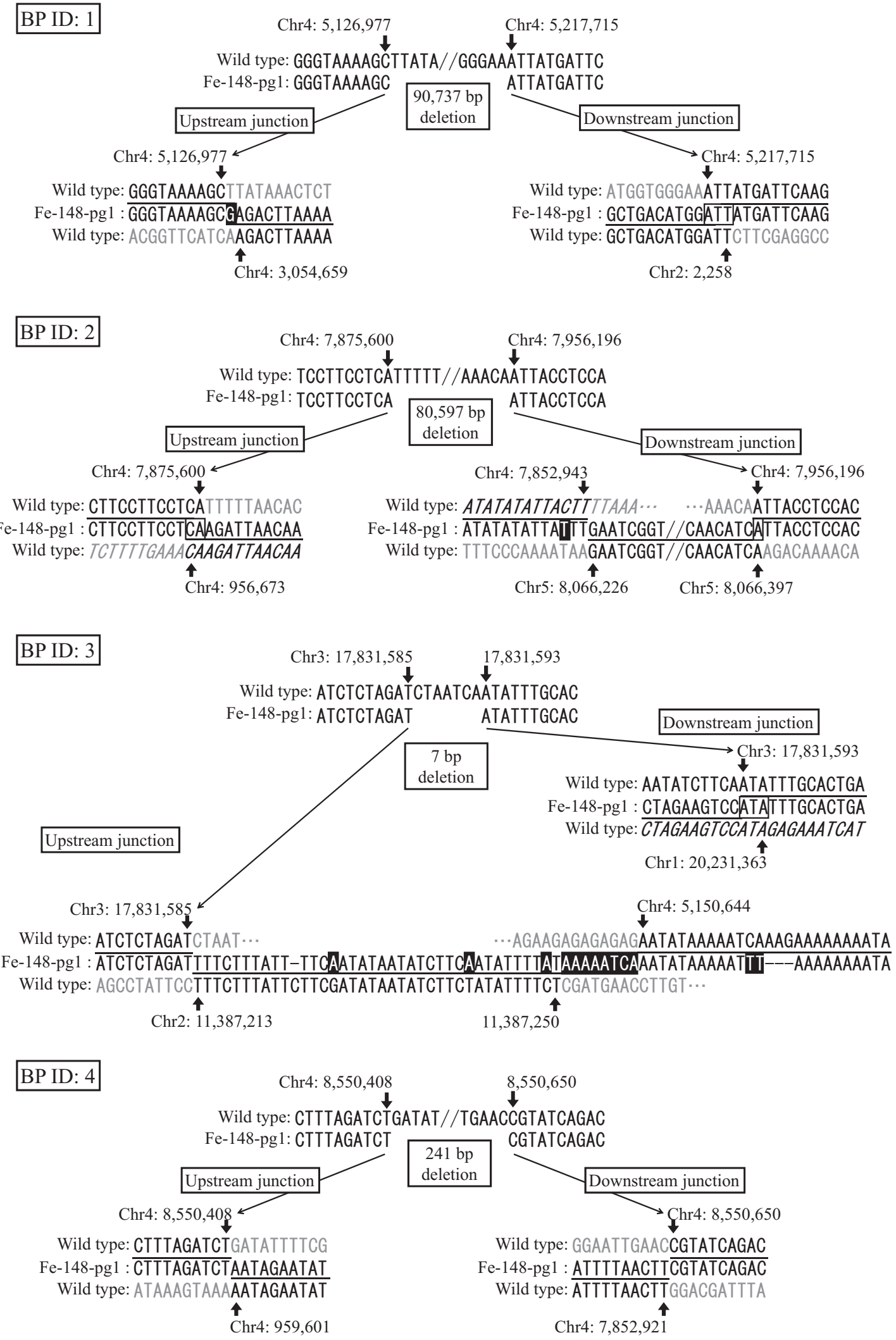

Fig. 3. Sequences of the rejoining sites in Fe-148-pg1. The break point (BP) IDs correspond to detected deletions that are represented in Table 2. The rejoined sites were identified using TAIL-PCR. In the sequences for the junctions, homologous regions are represented by lines that are drawn between the wild type and mutant sequences, and italics show the reversed sequences. The sequences in the black boxes represent base substitutions and inserted sequences, and micro-homologies in the rejoining sites are boxed. 
at 22.5-30.0 $\mathrm{keV} \cdot \mu^{-1}$, Kazama et al., 2011; $0 \%$ at 113 $\mathrm{keV} \cdot \mu \mathrm{m}^{-1}$, Shikazono et al., $2005 ; 8 \%$ at $290 \mathrm{keV} \cdot \mu \mathrm{m}^{-1}$, Hirano et al., 2012). Moreover, the 178-bp duplication that was detected in the Fe-88-nl1 mutant was not detected for any other type of heavy-ion irradiation with lower LETs; this duplication might have resulted from an error in synthesis-dependent strand annealing. Such large complexities at breakpoints appear to be reflected in the difficulty of repairing Fe-ion induced DNA lesions. We should note that the data on the deletion sizes in this study and previous ones could not be simply compared because the ways that they were analysed were different. Our results were obtained by analysing only 4 mutants, in which we detected breakpoints both in gene and nongene regions. On the other hand, previous results were obtained by analysing many mutants for which only one gene that was putatively responsible for their phenotypes was characterised.

\section{Array CGH can contribute to detecting deletions and duplications at the whole genome level Array} $\mathrm{CGH}$ is a well-developed technology for detecting changes in the copy number of chromosomal DNA segments (Halper-Stromberg et al., 2011). By using array CGH, we successfully detected deletions or duplications in an Fe-ion induced mutant (Fe-148-pg1). Our array design was thought to be able to detect deletions $200 \mathrm{bp}$ or larger, although unexpectedly, we could detect even a 7 bp deletion. Our data also demonstrated that our array design could detect both homologous and heterologous deletions. Thus, the array CGH could be used to determine the number of mutations at the whole genome level in which 200-bp deletions and more can be detected. Confirmation of these mutations by using PCR revealed that $\log _{2}$ ratios of over 1.2 should be considered as positive signals in this array platform, which indicates that deletions and duplications can be easily detected by using the $\log _{2}$ ratios as reliable indicators. The array CGH data in our experiment revealed that 7 deletions and 1 duplication were induced by Fe-ion irradiation at a dose of $50 \mathrm{~Gy}$. Such a small number of induced mutations could support the idea that heavy-ion beams are able to induce mutations to change a target trait without affecting other plant characteristics. This is thought to be due to the small number of induced mutations (Abe et al., 2012). Further experimental evidence is needed to confirm this issue.

\section{CONCLUSIONS}

In the present study, we revealed that Fe-ion beam can induce a large variety of mutations, such as deletions, duplications, insertions, and translocations. In particular, a combination of large deletions and chromosomal rearrangements was detected by using a PCR-based method and array CGH. Therefore, Fe-ion irradiation could be useful for the study of chromosome rearrangements or knockouts of tandemly arrayed genes. Moreover, the combination of Fe-ion irradiation and array $\mathrm{CGH}$ within our design is a valuable method for screening rearrangements at the whole genome level. We should note that Fe-ion irradiation also causes small mutations such as 2-bp deletions. Therefore, genome re-sequencing of mutants by using next generation sequencing is needed to completely clarify the effect of heavy-ion irradiation on the whole genome.

We thank the RIKEN Research Resources Center of the Brain Science Institute for performing DNA sequencing. This experiment was performed at RIBF operated by the RIKEN Nishina Center and CNS, University of Tokyo. This research was supported by a grant from the Japan Society for the Promotion of Science (JSPS) to TA through the "Funding Program for Next Generation World-Leading Researchers (NEXT Program)", which was initiated by the Council for Science and Technology Policy (CSTP). This research was also partially supported by JSPS KAKENHI (Grant No. 23770070 and 25292009 to YK, and 22780034 to TH), the Special Postdoctoral Research Program of RIKEN (to YK), and by an Incentive Research Grant from RIKEN (to YK).

\section{REFERENCES}

Abe, T., Ryuto, H., and Fukunishi, N. (2012) Ion beam radiation mutagenesis. In: Plant Mutation Breeding and Biotechnology (eds.: Q. Y. Shu, B. P. Forster, and H. Nakagawa), pp. 99106. CABI, Oxford.

Alloni, D., Campa, A., Belli, M., Esposito, G., Facoetti, A., Friedland, W., Liotta, M., Mariotti, L., Paretzke, H. G., and Ottolenghi, A. (2010) A Monte Carlo study of the radiation quality dependence of DNA fragmentation spectra. Radiat. Res. 173, 263-271.

Blocher, D. (1988) DNA double-strand break repair determines the RBE of alpha-particles. Int. J. Radiat. Biol. 54, 761-771.

Fakir, H., Sachs, R. K., Stenerlow, B., and Hofmann, W. (2006) Clusters of DNA double-strand breaks induced by different doses of nitrogen ions for various LETs: experimental measurements and theoretical analyses. Radiat. Res. 166, 917927.

Fujita, N., Torii, C., Ishii, K., Aonuma, W., Shimizu, Y., Kazama, Y., Abe, T., and Kawano, S. (2012) Narrowing down the mapping of plant sex-determination regions by using new Ychromosome-specific markers and heavy-ion beam irradiation-induced Y-deletion mutants in Silene latifolia. G3 2 , 271-278.

Gorbunova, V., and Levy, A. A. (1999) How plants make ends meet: DNA double-strand break repair. Trends Plant Sci. 4, 263-269.

Halper-Stromberg, E., Frelin, L., Ruczinski, I., Scharpf, R., Jie, C., Carvalho, B., Hao, H., Hetrick, K., Jedlicka, A., Dziedzic, A., et al. (2011) Performance assessment of copy number microarray platforms using a spike-in experiment. Bioinformatics 27, 1052-1060.

Hase, Y., Tanaka, A., Baba, T., and Watanabe, H. (2000) FRL1 is required for petal and sepal development in Arabidopsis. Plant J. 24, 21-32.

Hase, Y., Trung, K. H., Matsunaga, T., and Tanaka, A. (2006) A mutation in the uvi4 gene promotes progression of endoreduplication and confers increased tolerance towards ultra- 
violet B light. Plant J. 46, 317-326.

Hirano, T., Kazama, Y., Ohbu, S., Shirakawa, Y., Liu, Y., Kambara, T., Fukunishi, N., and Abe, T. (2012) Molecular nature of mutations induced by high-LET irradiation with argon and carbon ions in Arabidopsis thaliana. Mutat. Res. 735, 19-31.

Hirano, T., Takagi, K., Hoshino, Y., and Abe, T. (2013) DNA damage response in male gametes of Cyrtanthus mackenii during pollen tube growth. AoB Plants 5, plt004.

Horiguchi, G., Kim, G. T., and Tsukaya, H. (2005) The transcription factor AtGRF5 and the transcription coactivator AN3 regulate cell proliferation in leaf primordia of Arabidopsis thaliana. Plant J. 43, 68-78.

Ichida, H., Matsuyama, T., Ryuto, H., Hayashi, Y., Fukunishi, N., Abe, T., and Koba, T. (2008) Molecular characterization of microbial mutations induced by ion beam irradiation. Mutat. Res. 639, 101-107.

Ishikawa, S., Ishimaru, Y., Igura, M., Kuramata, M., Abe, T., Senoura, T., Hase, Y., Arao, T., Nishizawa, N. K., and Nakanishi, H. (2012) Ion-beam irradiation, gene identification, and marker-assisted breeding in the development of low-cadmium rice. Proc. Natl. Acad. Sci. USA 109, 1916619171.

Kazama, Y., Saito, H., Yamamoto, Y. Y., Hayashi, Y., Ichida, H., Ryuto, H., Fukunishi, N., and Abe, T. (2008) LET-dependent effects of heavy-ion beam irradiation in Arabidopsis thaliana. Plant Biotechnol. 25, 113-117.

Kazama, Y., Hirano, T., Saito, H., Liu, Y., Ohbu, S., Hayashi, Y., and Abe, T. (2011) Characterization of highly efficient heavy-ion mutagenesis in Arabidopsis thaliana, BMC Plant Biol. 11, 161.

Kazama, Y., Ma, L., Hirano, T., Ohbu, S., Shirakawa, Y., Hatakeyama, S., Tanaka, S., and Abe, T. (2012) Rapid evaluation of effective linear energy transfer in heavy-ion mutagenesis of Arabidopsis thaliana. Plant Biotechnol. 29, 441445.

Kazama, Y., Fujiwara, M. T., Takehisa, H., Ohbu, S., Saito, H., Ichida, H., Hayashi, Y., and Abe, T. (2013) Characterization of a heavy-ion induced white flower mutant of allotetraploid Nicotiana tabacum. Plant Cell Rep. 32, 11-19.

Kim, G. T., Shoda, K., Tsuge, T., Cho, K. H., Uchimiya, H., Yokoyama, R., Nishitani, K., and Tsukaya, H. (2002) The ANGUSTIFOLIA gene of Arabidopsis, a plant CtBP gene, regulates leaf-cell expansion, the arrangement of cortical microtubules in leaf cells and expression of a gene involved in cell-wall formation. EMBO J. 21, 1267-1279.

Koornneef, M., Rolff, E., and Spruit, C. J. P. (1980) Genetic control of light-inhibited hypocotyl elongation in Arabidopsis thaliana (L) Heynh. Z. Pflanzenphysiol. 100, 147-160.

Koornneef, M., Dellaert, L. W. M., and van der Veen, J. H. (1982) EMS- and radiation-induced mutation frequencies at individual loci in Arabidopsis thaliana (L.) Heynh. Mutat. Res. 93, 109-123.

Liu, Y. G., Mitsukawa, N., Oosumi, T., and Whittier, R. F. (1995) Efficient isolation and mapping of Arabidopsis thaliana TDNA insert junctions by thermal asymmetric interlaced PCR. Plant J. 8, 457-463.

Lucito, R., Healy, J., Alexander, J., Reiner, A., Esposito, D., Chi, M., Rodgers, L., Brady, A., Sebat, J., Troge, J., et al. (2003) Representational oligonucleotide microarray analysis: a high-resolution method to detect genome copy number variation. Genome Res. 13, 2291-2305.

Ma, L., Kazama, Y., Inoue, H., Abe, T., Hatakeyama, S., and Tanaka, S. (2013) The type of mutations induced by carbonion-beam irradiation of the filamentous fungus Neurospora crassa. Fungal Biol. 117, 227-238.

Morita, R., Kusaba, M., Iida, S., Yamaguchi, H., Nishio, T., and Nishimura, M. (2009) Molecular characterization of mutations induced by gamma irradiation in rice, Genes Genet. Syst. 84, 361-370.

Nagano, A. J., Fukazawa, M., Hayashi, M., Ikeuchi, M., Tsukaya, H., Nishimura, M., and Hara-Nishimura, I. (2008) AtMap1: a DNA microarray for genomic deletion mapping in Arabidopsis thaliana. Plant J. 56, 1058-1065.

Naito, K., Kusaba, M., Shikazono, N., Takano, T., Tanaka, A., Tanisaka, T., and Nishimura, M. (2005) Transmissible and nontransmissible mutations induced by irradiating Arabidopsis thaliana pollen with $\gamma$-rays and carbon ions. Genetics 169, 881-889.

Pastwa, E., Neumann, R. D., Mezhevaya, K., and Winters, T. A. (2003) Repair of radiation-induced DNA double-strand breaks is dependent upon radiation quality and the structural complexity of double-strand breaks. Radiat. Res. 159, 251-261.

Sasaki, K., Yamaguchi, H., Aida, R., Shikata, M., Abe, T., and Ohtsubo, N. (2012) Mutation in Torenia fournieri Lind. $U F O$ homolog confers loss of $T f L F Y$ interaction and results in a petal to sepal transformation. Plant J. 71, 1002-1014.

Shikazono, N., Suzuki, C., Kitamura, S., Watanabe, H., Tano, S., and Tanaka, A. (2005) Analysis of mutations induced by carbon ions in Arabidopsis thaliana. J. Exp. Bot. 56, 587-596.

Shimada, S., Ogawa, T., Kitagawa, S., Suzuki, T., Ikari, C., Shitsukawa, N., Abe, T., Kawahigashi, H., Kikuchi, R., Handa, H., and Murai, K. (2009) A genetic network of flowering-time genes in wheat leaves, in which an APETALA1/ FRUITFULL-like gene, VRN1, is upstream of FLOWERING LOCUS T. Plant J. 58, 668-681.

Shitsukawa, N., Ikari, C., Shimada, S., Kitagawa, S., Sakamoto, K., Saito, H., Ryuto, H., Fukunishi, N., Abe, T., Takumi, S., Nasuda, S., and Murai, K. (2007) The einkorn wheat (Triticum monococcum) mutant, maintained vegetative phase, is caused by a deletion in the VRN1 gene. Genes Genet. Syst. 82, 167-170.

Tanaka, A., Shikazono, N., and Hase, Y. (2010) Studies on biological effects of ion beams on lethality, molecular nature of mutation, mutation rate, and spectrum of mutation phenotype for mutation breeding in higher plants. J. Radiat. Res. 51, 223-233.

Venglat, S. P., Dumonceaux, T., Rozwadowski, K., Parnell, L., Babic, V., Keller, W., Martienssen, R., Selvaraj, G., and Datla, R. (2002) The homeobox gene BREVIPEDICELLUS is a key regulator of inflorescence architecture in Arabidopsis. Proc. Natl. Acad. Sci. USA 99, 4730-4735.

Walker, A. R., Davison, P. A., Bolognesi-Winfield, A. C., James, C. M., Srinivasan, N., Blundell, T. L., Esch, J. J., Marks, M. D., and Gray, J. C. (1999) The TRANSPARENT TESTA GLABRA1 locus, which regulates trichome differentiation and anthocyanin biosynthesis in Arabidopsis, encodes a WD40 repeat protein. Plant Cell 11, 1337-1349.

Yasui, Y., Mori, M., Aii, J., Abe, T., Matsumoto, D., Sato, S., Hayashi, Y., Ohnishi, O., and Ota, T. (2012) S-LOCUS EARLY FLOWERING 3 is exclusively present in the genomes of short-styled buckwheat plants that exhibit heteromorphic self-incompatibility. PLoS One 7, e31264.

Yatagai, F. (2004) Mutations induced by heavy charged particles. Biol. Sci. Space 18, 224-234.

Yu, P., Wang, C., Xu, Q., Feng, Y., Yuan, X., Yu, H., Wang, Y., Tang, S., and Wei, X. (2011) Detection of copy number variations in rice by using array-based comparative genomic hybridization. BMC Genomics 12, 372. 